\title{
Standard vs delayed ligature of the dorsal vascular complex during robot-assisted radical prostatectomy: results from a randomized controlled trial
}

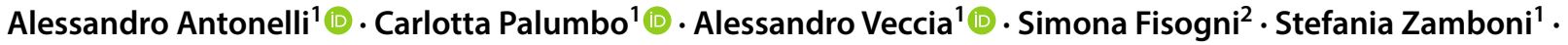 \\ Maria Furlan $^{1}$ - Simone Francavilla ${ }^{1}$ ( ) Marco Lattarulo ${ }^{1}$ - Enrico De Marzo ${ }^{1}$. Giuseppe Mirabella ${ }^{1}$. Angelo Peroni ${ }^{1}$. \\ Claudio Simeone ${ }^{1}$ (1)
}

Received: 21 May 2018 / Accepted: 9 July 2018

(c) Springer-Verlag London Ltd., part of Springer Nature 2018

\begin{abstract}
Purpose Prospective randomized trial to compare standard vs delayed approach to dorsal vascular complex (s-DVC vs d-DVC) in robot-assisted radical prostatectomy (RARP).

Methods Patients scheduled for RARP were randomized into a 1:1 ratio to receive either s-DVC or d-DVC by two experienced surgeons. In s-DVC arm an eight-shaped single stitch was given at the beginning of the procedure and the DVC was subsequently cut at time of apical dissection; in d-DVC arm the plexus was transected at the end of prostatectomy, prior to apex dissection and then sutured. Primary endpoint was difference in estimated blood loss (EBL) and a sample size of 226 cases was calculated; ad interim analysis was planned after $2 / 3$ of recruitment.

Results Endpoint was reached at ad interim analysis after 162 cases ( $81 \mathrm{~s}-\mathrm{DVC}, 81 \mathrm{~d}-\mathrm{DVC}$ ) and recruitment was, therefore, interrupted. Baseline and tumor characteristics were overlapping. EBL was significantly higher in d-DVC arm (mean EBL 107 vs $65 \mathrm{ml}, p=0.003$ ), but without differences in post-operative hemoglobin, transfusions and complications. Overall PSM rate was higher in d-DVC arm (21.0 vs $14.8 \%, p=0.323)$, with statistical significance relatively to organ-confined disease $(15.5$ vs $3.6 \%, p=0.031)$. Apical involvement was instead significantly higher in s-DVC arm (prevalence in PSM patients 66.7 vs $23.5 \%, p=0.020$ ). Post-operative PSA, continence and potency rates were similar between groups.

Conclusions Standard and delayed approaches to DVC are safe and lead to similar functional outcomes. A delayed approach exposes to a higher risk of PSM in organ-confined disease but with a lower risk of apical involvement.
\end{abstract}

Keywords Dorsal vascular complex $\cdot$ Robot-assisted radical prostatectomy $\cdot$ Prostate cancer $\cdot$ Blood loss $\cdot$ Positive surgical margins

\section{Introduction}

The original description of open retropubic retrograde radical prostatectomy by Walsh and Lepor [1] prescribed to ligate the dorsal vascular complex (DVC) during the first steps of the procedure, immediately before to proceed for apical dissection. This maneuver aims at to control possible

Carlotta Palumbo

palumbo.carlotta@gmail.com

1 Department of Urology, Spedali Civili Hospital, University of Brescia, Piazzale Spedali Civili 1, 25123 Brescia, Italy

2 Department of Pathology, Spedali Civili Hospital, University of Brescia, Piazzale Spedali Civili 1, 25123 Brescia, Italy prohibitive bleeding that could worsen peri-operative course and impair both functional and oncological outcomes, given the strict anatomical relationships between DVC, prostatic apex, striated sphincter and neurovascular bundles [2].

Since a decade, robot-assisted radical prostatectomy (RARP) has challenged open prostatectomy as standard surgical approach for prostate cancer, even if its superiority is still under debate [3-7]. Despite the anterograde-instead of retrograde - way of dissection robotics inherited from open prostatectomy the way to deal with the DVC: an early ligature of DVC and its subsequent transection has indeed been generally considered as the standard approach to the DVC. However, it was postulated that suturing the plexus prior to apical dissection could tether the sphincter or prostatic tissue and render more challenging to develop the correct plan of 
dissection during apical detachment. Consequently, various technical modifications of minimally-invasive radical prostatectomy have been proposed counting on the hemostatic effect exerted by the intra-abdominal pressure given by the pneumoperitoneum $[8,9]$. The most accepted alternative approach consists of the direct section of the DVC at the end of prostatectomy, going for suture afterwards: this sequence of steps is generally denominated delayed approach to the DVC.

The only available methodologically sound comparison between these two options has been published by Porpiglia and coll, that tested standard vs delayed approach in a randomized fashion in purely laparoscopic radical prostatectomies [10] showing an advantage on continence recovery for the latter. Nevertheless, the translation of these results to RARP is debatable because $3 \mathrm{D}$ vision and the intuitiveness and articulation of instruments should favor robotics, specifically in suturing. At least from a theoretically point of view, the better quality of sutures provided by robotic systems should be beneficial for both the approaches, either making preliminary stitch more selective or facilitating suturing after the transection of DVC.

During scientific meetings and live surgery events, an endless debate on the pro and contra of the different ways to manage DVC in RARP occurs, but this issue has been taken into account only by a few retrospective studies [11-13]. So, in the lack of high-quality evidence, these two alternatives are chosen just on personal preferences and beliefs of surgeon.

Thus, we designed a prospective randomized controlled trial to compare standard (s-DVC) vs delayed approach to DVC (d-DVC) to assess differences in surgical, oncological and functional outcomes.

\section{Materials and methods}

\section{Study design and population}

A phase III prospective randomized controlled trial was designed in adherence to the CONSORT (CONsolidated Standards of Reporting Trials) guidelines [14] to compare standard (s-DVC group) with delayed (d-DVC group) ligation of DVC. Authorization from the Institutional Review Board and Ethical Committee of Spedali Civili Hospital was obtained in August 2016 (approval number NP 2400) and registered at clinicaltrial.gov (identifier NCT03351088).

All consecutive patients scheduled for RARP were considered eligible. Inclusion criteria were patients aged 18 or older, willing and able to provide informed consent and histological diagnosis of prostate cancer for whom radical prostatectomy was indicated. Exclusion criteria were previous pelvic radiotherapy, impairment of coagulation or participation in other interventional trials.

Allocation concealment was ensured by the creation of a password-protected randomization database, generated by an automatic algorithm (http://www.randomizer.org); randomization was done on a 1:1 ratio without stratification. All information was registered into a dedicated database. According to institutional policy anti-platelets agents were not suspended, while oral anticoagulation drugs were replaced by low-molecular weight heparin. A neoadjuvant course of androgen-deprivation therapy was administered at the discretion of the referring urologist. Comorbidities were summarized by the Charlson-Romano index [15] and American Society of Anesthesiology score [16]; operative time was calculated from skin incision to the closure of all wounds; estimated blood loss (EBL) was intended as the blood volume collected into the suctioning system. Lymph node dissection was indicated when the risk of lymph node invasion overcame 5\% according to Briganti's nomogram [17]. A nerve-sparing procedure was generally performed in low/intermediate-risk PCa according to D'Amico classification [18], bi- or unilaterally according to the risk of neurovascular invasion at each side as indicated by clinical framework or intra-operative view. A drainage was regularly put and removed when clinically indicated, generally at the recovery of bowel canalization; the catheter was generally maintained for 7 days, and removed without cystogram, if not clinically indicated. 30-day complications were prospectively recorded in according to Clavien-Dindo grading system through face-to-face interview with a medical doctor $[19,20]$. All specimens were evaluated by an expert uropathologist (SF) following International Society of Uropathologist recommendations [21]. Positive surgical margins (PSM) were defined as the presence of cancer cells on the inked surface of prostate and sites of positivity were detailed. Follow-up controls were done at a dedicated outpatient office, according to internal schedule (visit and PSA at 1,3 and 6 months for 2 years, and then yearly) and included the administration of validated questionnaires, as The Expanded Prostate Cancer Index Composite (EPIC) [22].

\section{Surgical technique}

Robotics was introduced at our institution since 2010 with the acquisition of a four-arm DaVinci ${ }^{\circledR} \mathrm{Si}$ system (Intuitive Surgical, Inc. Sunnyvale, CA, USA) and nowadays it is our exclusive approach for radical prostatectomy. Two surgeons performed all robotic procedures and at the time of the study achieved equivalent experience of around 350 RARP. The procedure was done through a transperitoneal anterograde approach, broadly following the technique of Patel and coll [23]. A so-called "urethral sparing" was generally attempted during apical dissection, unless the risk of cancer 
involvement was deemed as significant, due to positive biopsies and/or clinical, radiological or intra-operative suspicion. For all the procedures, thermal energy was applied by pinpoint application of low-intensity $(25 \mathrm{~W})$ mono-polar cautery and omitted, as much as possible, during neurovascular and apical dissection. Non-readsorbable clips (Hem-o-lok ${ }^{\circledR}$ ligation system, Weck, Teleflex, USA) were used only on prostatic pedicles.

Per protocol, in the standard ligation arm (s-DVC), after opening the endopelvic fascia, the lateral aspects of prostate were dissected and the DVC isolated and sutured by a $1-0$ monofilament suture with a $1 / 2$ circle 40 -mm needle, avoiding to throttle the pubo-prostatic ligaments (Fig. 1).

In the delayed ligation arm (d-DVC), the DVC was thermally incised immediately before apex dissection; once prostate detachment was completed, a vertical running suture with 3-0 monofilament on a $5 / 8$ circle $26-\mathrm{mm}$ needle was done, side-to-side, right to left (Fig. 2); in case of bleeding intra-abdominal pressure was increased up to $15-18 \mathrm{mmHg}$ and suction by the assistant discouraged.

\section{Endpoints}

The primary endpoint was estimated blood loss (EBL): this choice aimed at to warrant the feasibility and reproducibility of the study, both in statistical and clinical terms. Indeed the few existing literature on the outcomes of s- vs d-DVC approaches for RARP reports consistent results only with regard to EBL. Moreover, the planned difference between arms based on EBL allowed a sample size affordable for the case load of our institution. Other outcomes of prominent clinical interest were instead adopted as secondary endpoints and were overall and apical positive surgical margins (PSM), 1-month PSA value, and continence and potency rates. In particular, continence was defined as "no need for pads" or "1 security pad/day" (EPIC question number 5 "How many pads or adult diapers per day did you usually use to control leakage during the last 4 weeks?" [22]), while potency as the ability to achieve intercourse spontaneously or with oral drugs.

\section{Sample size definition}

In our previous experience with s-DVC approach a mean EBL of $54.0 \mathrm{ml}$ (standard deviation, $\mathrm{SD} \pm 75.3 \mathrm{ml}$ ) was observed. The few published studies on this issue [11-13] reported that d-DVC approach determined a positive difference in EBL, ranging from 10 to $100 \mathrm{ml}$. Therefore, for the present trial we arbitrarily set a difference of $30 \mathrm{ml}$ as significant, because equal to a relative increase $>50 \%$ with respect to our historical data and within the range reported by the literature. Considering an expected EBL of $54.0 \pm 75.3 \mathrm{ml}$ for s-DVC, and a difference of $30 \mathrm{ml}$ in d-DVC, the sample size-estimated by log-rank test of equal proportions with an $\alpha$ of 0.05 and a power of $85 \%$-was 226 cases, 113 for each arm. An ad interim analysis was planned at two-third of enrollment, namely 150 patients.
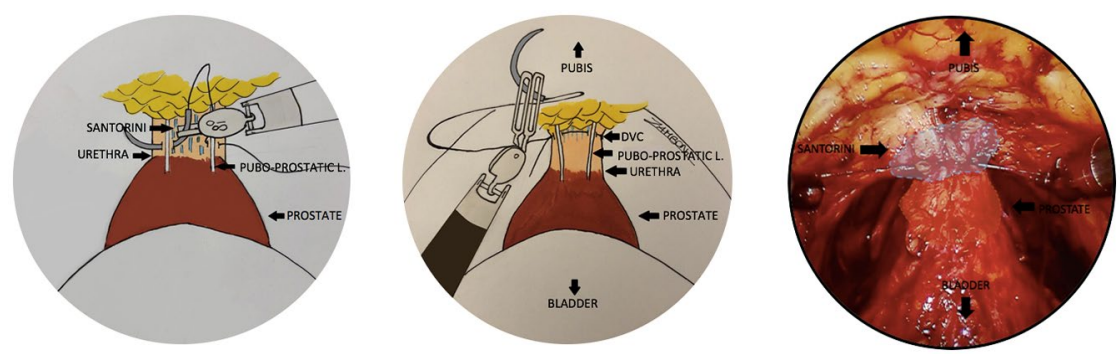

Fig. 1 Standard ligation of DVC: after opening the endopelvic fascia and lateral dissection of prostate up to the apex, a eight-figure 1-0 CT-1 stitch was given, without including pubo-prostatic ligaments; transection of the DVC was postponed at the end of prostatectomy before the section of the urethra
Fig. 2 Delayed ligation of DVC: at the end of prostatectomy, prior to apex dissection, the DVC was transected; once completed the detachment of prostate, a vertical running suture with 3-0 UR-6 needle, side-to-side, right to left, was given
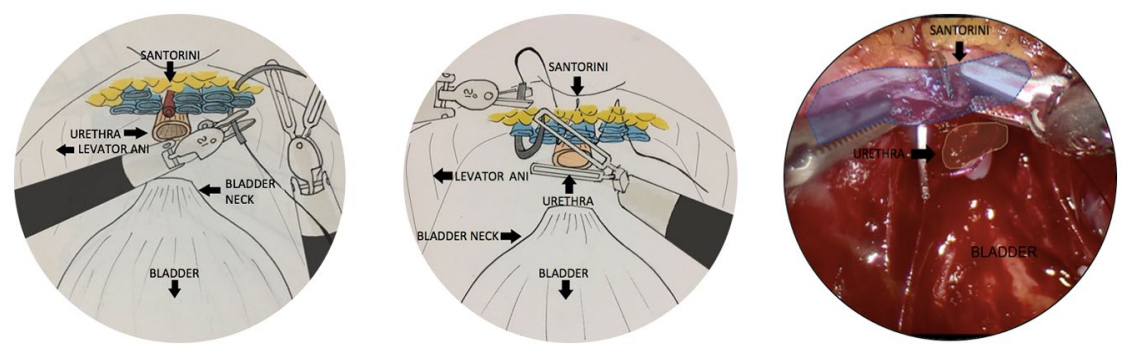


\section{Statistical analysis}

Variables were reported as median \pm standard deviation (SD) for parametric distribution, median and interquartile range (IQR) for non-parametric, numbers and proportions for categorical variables. Differences were compared using the Student $t$-test, Mann-Whitney u-test, Pearson chi-square or Fisher exact test, as appropriate. Data were analyzed according to a per protocol principle, including only patients in whom no protocol violation occurred. Differences were considered significant for $p<0.05$; all tests were two sided. Analysis was performed with Stata software v14.0 (StataCorp LLC, TXS, USA).

\section{Results}

\section{Baseline features}

From August 2016 to December 2017, 162 patients were enrolled and randomized for s-DVC (81 patients) vs d-DVC arms (81 patients). At the time of planned ad interim analysis, the difference in EBL between arms was statistically significant (s-DVC vs d-DVC, mean EBL 65 vs $107 \mathrm{ml}$, $p=0.003$, difference $42 \mathrm{ml}$ ), so that enrollment was suspended and the analysis finalized.

Baseline features of both groups were overlapping, in particular regarding the clinical and bioptic features of $\mathrm{PCa}$ (Table 1).
Table 1 Baseline features of cohort

\begin{tabular}{|c|c|c|c|}
\hline & s-DVC & d-DVC & $p$ value \\
\hline Age (years), mean $( \pm \mathrm{SD})$ & $64.2( \pm 6.4)$ & $65.0( \pm 6.4)$ & 0.385 \\
\hline $\mathrm{BMI}(\mathrm{kg} / \mathrm{m} 2)$, mean $( \pm \mathrm{SD})$ & $26.4( \pm 3.6)$ & $26.4( \pm 3.5)$ & 0.991 \\
\hline Charlson Comorbidity Index, median (IQR) & $0(0-1)$ & $0(0-1)$ & 0.567 \\
\hline ASA score, median (IQR) & $2(2-2)$ & $2(2-2)$ & 0.929 \\
\hline Antiplatelet/Anticoagulant therapy, number (\%) & $14(17.3 \%)$ & $17(21.2 \%)$ & 0.523 \\
\hline Previous TURP, number (\%) & $3(3.7 \%)$ & $3(3.7 \%)$ & 1.000 \\
\hline Alfa-blockers, number $(\%)$ & $19(23.5 \%)$ & $17(21.0 \%)$ & 0.705 \\
\hline 5-alfa reductase inhibitors, number $(\%)$ & $5(6.2 \%)$ & $5(6.2 \%)$ & 1.000 \\
\hline Positive digital rectal examination, number (\%) & $16(19.7 \%)$ & $24(29.6 \%)$ & 0.145 \\
\hline TRUS prostate volume $(\mathrm{cc})$, mean $( \pm \mathrm{SD})$ & $48.9 \pm 24.5$ & $48.3 \pm 27.2$ & 0.879 \\
\hline Pre-operative PSA $(\mathrm{ng} / \mathrm{mL})$, mean $( \pm \mathrm{SD})$ & $9.1( \pm 13.1)$ & $8.2( \pm 5.7)$ & 0.594 \\
\hline Positive cores (number), median (IQR) & $3(2-5)$ & $4(2-6)$ & 0.366 \\
\hline \multicolumn{4}{|l|}{ Core's involvement $(\%)$, mean $( \pm \mathrm{SD})$} \\
\hline Mean involvement & $22.2 \pm 17.5$ & $27.0 \pm 20.1$ & 0.254 \\
\hline Maximum involvement & $36.0 \pm 28.7$ & $43.1 \pm 28.8$ & 0.134 \\
\hline Clinical stage, number $(\%)$ & & & 0.941 \\
\hline $\mathrm{cT} 1$ & $64(79.0 \%)$ & $60(74.1 \%)$ & \\
\hline $\mathrm{cT} 2$ & $15(18.5 \%)$ & $19(23.4 \%)$ & \\
\hline cT3 & $2(2.5 \%)$ & $2(2.5 \%)$ & \\
\hline Biopsy ISUP group, number (\%) & & & 0.586 \\
\hline 1 & $38(47.0 \%)$ & $41(50.5 \%)$ & \\
\hline 2 & $22(27.2 \%)$ & $18(22.2 \%)$ & \\
\hline 3 & $10(12.2 \%)$ & $14(17.3 \%)$ & \\
\hline 4 & $7(8.6 \%)$ & $4(5.0 \%)$ & \\
\hline 5 & $4(5.0 \%)$ & $4(5.0 \%)$ & \\
\hline D’Amico risk class, number (\%) & & & 0.596 \\
\hline Low risk & $32(39.5 \%)$ & $35(43.2 \%)$ & \\
\hline Intermediate risk & $38(46.9 \%)$ & $39(48.1 \%)$ & \\
\hline High risk & $11(13.6 \%)$ & $7(8.6 \%)$ & \\
\hline Neoadjuvant androgen-deprivation therapy, number (\%) & $18(22.2 \%)$ & $18(22.2 \%)$ & 1.000 \\
\hline
\end{tabular}

$S D$ standard deviation, $B M I$ Body Mass Index, IQR interquartile range, ASA American Society of Anesthesiology, TURP transurethral resection of the prostate, PSA prostate-specific antigen, ISUP international society of uropathologist 


\section{Intra-operative data and peri-operative outcomes}

Groups were balanced concerning surgeon, lymphadenectomy, nerve-sparing dissection and operative time. As above reported a significant difference was found in EBL, although post-operative decrease in hemoglobin value was similar $(-1.5 \mathrm{~g} / \mathrm{dl}$ for each arm), as well as for the days of drainage and catheter, transfusions (1 event in d-DVC group) and complications in general (1 ureteral lesion Clavien-Dindo $3 \mathrm{~b}$, requiring robotic reimplantation in 14 th post-operative day in d-DVC group) (Table 2).

\section{Oncological outcomes}

The characteristics of PCa at final pathology were equivalent between groups regarding tumor volume, local staging, lymph node invasion and grading. Also overall PSM rate was similar (d-DVC vs s-DVC 21.0 vs $14.8 \%$, $p=0.323$ ), but subgroup analysis showed a significant higher PSM rate for the d-DVC group in case of pT2 disease (15.5 vs $3.6 \%, p=0.031)$. The topography of the sites of PSM was similar, except for the location at the apex that was significantly more frequent in the s-DVC group, representing 66.7 vs $23.5 \%$ of PSMs ( $p=0.020)$. Post-operative PSA at 1, 3 and 6 months was available for 162, 116 and 82 patients, respectively, and was similar between s-DVC and d-DVC groups (Table 3). Only four patients (one in s-DVC vs three in d-DVC, $p$ not significant) experienced a biochemical relapse.

\section{Functional outcomes}

Continence was assessed at 1, 3 and 6 months finding similar rates between s-DVC and d-DVC, equal to 81.5 vs $76.5 \%$ $(p=0.440), 95.0$ vs $91.4 \%(p=0.393)$ and 97.0 vs $98.0 \%$ $(p=0.752)$, respectively. Using the definition "no pads" these figures declined to 39.5 vs $40.7 \%(p=0.873), 63.3$ vs $60.0 \%(p=0.680)$ and $73.1 \mathrm{vs} 77.5 \%(p=0.587)$. Also the potency rate, evaluated in 88 patients submitted to a nervesparing procedure, was similar between s-DVC and d-DVC and equal to 28.3 vs $21.4 \%(p=0.460)$ at 1 month, 37.8 vs $31.6 \%(p=0.555)$ at 3 months and 50.0 vs $44.8 \%(p=0.668)$ at 6 months.

\section{Discussion}

The present trial aims at giving evidence useful to shed light on the debate on which is the preferential approach to the DVC during RARP. In summary, the main findings are: (1) intra- and peri-operative courses overlapped and this corroborates the safety of the delayed approach; (2) the recovery of continence and potency occurred at similar times and magnitude, confuting the advantages advocated for the delayed approach; (3) incidence and topographic distribution of PSMs were different, with higher PSM rate in pT2 cancer, but also a lower prevalence of apex involvement in d-DVC.

Despite the randomized and prospective design of the present study, these findings deserve a careful and in-depth critical discussion. Unfortunately, the room for a comparison
Table 2 Intra-operative features and peri-operative outcomes

\begin{tabular}{llll}
\hline & s-DVC & d-DVC & $p$ value \\
\hline First surgeon, number of procedures $(\%)$ & & & 0.875 \\
1 & $39(48.1 \%)$ & $40(49.3 \%)$ & \\
2 & $42(51.8 \%)$ & $41(50.6 \%)$ & \\
Lymph node dissection, number (\%) & $32(39.5 \%)$ & $34(42.0 \%)$ & 0.749 \\
Nerve-sparing procedure, number (\%) & $46(56.8 \%)$ & $42(51.8 \%)$ & 0.528 \\
Bilateral nerve-sparing procedure, number $(\%)$ & $31(38.2 \%)$ & $27(33.3 \%)$ & 0.888 \\
Operative time (minutes), mean $( \pm \mathrm{SD})$ & $205.6( \pm 37.2)$ & $200.2( \pm 39.2)$ & 0.398 \\
Estimated blood loss (mL), mean $( \pm \mathrm{SD})$ & $65.0( \pm 96.6)$ & $107.1( \pm 134.2)$ & 0.003 \\
Post-operative transfusion, number $(\%)$ & $0(0 \%)$ & $1(1.2 \%)$ & 0.313 \\
Delta pre-operative/3rd POD Hb (g/dl), mean $( \pm \mathrm{SD})$ & $-1.5( \pm 1.4)$ & $-1.5( \pm 1.1)$ & 0.787 \\
Clavien-Dindo post-operative complications, number $(\%)$ & & & 0.428 \\
0 & $79(97.5 \%)$ & $75(92.6 \%)$ & \\
1 & $2(2.5 \%)$ & $4(5.0 \%)$ & \\
2 & $0(0 \%)$ & $1(1.2 \%)$ & \\
$3 \mathrm{~b}$ & $0(0 \%)$ & $1(1.2 \%)$ & \\
Days of drainage, median (IQR) & $3(2-4)$ & $3(2-3)$ & 0.299 \\
Day of feeding, median (IQR) & $2(1-2)$ & $2(1-2)$ & 0.878 \\
Days of catheter, median (IQR) & $7(6-7)$ & $7(7-7)$ & 0.174 \\
\hline
\end{tabular}

$S D$ standard deviation, $P O D$ post-operative day 
Table 3 Pathological findings and oncological outcomes

\begin{tabular}{|c|c|c|c|}
\hline & s-DVC & d-DVC & $p$ value \\
\hline Pathological T stage, number (\%) & & & 0.643 \\
\hline $\mathrm{pT} 2 \mathrm{a}$ & $7(8.6 \%)$ & $8(9.9 \%)$ & \\
\hline pT2b & $4(4.9 \%)$ & $1(1.2 \%)$ & \\
\hline pT2c & $46(56.8 \%)$ & $49(60.5 \%)$ & \\
\hline pT3a & $19(23.5 \%)$ & $15(18.5 \%)$ & \\
\hline pT3b & $2(2.5 \%)$ & $5(6.2 \%)$ & \\
\hline $\mathrm{pN}+$ & $3(3.7 \%)$ & $3(3.7 \%)$ & \\
\hline Pathological ISUP group, number (\%) & & & 0.426 \\
\hline 1 & $32(39.5 \%)$ & $28(34.6 \%)$ & \\
\hline 2 & $16(19.7 \%)$ & $22(27.2 \%)$ & \\
\hline 3 & $11(13.6 \%)$ & $11(13.6 \%)$ & \\
\hline 4 & $5(6.2 \%)$ & $1(1.2 \%)$ & \\
\hline 5 & $9(11.1 \%)$ & $9(11.1 \%)$ & \\
\hline Not assessable & $8(9.9 \%)$ & $10(12.3 \%)$ & \\
\hline Tumor volume (\% on whole gland), median (IQR) & $8(5-15)$ & $8(5-16)$ & 0.902 \\
\hline \multicolumn{4}{|l|}{ PSM analysis } \\
\hline PSM overall, number ( $\%$ on 162 patients) & $12(14.8 \%)$ & $17(21.0 \%)$ & 0.323 \\
\hline PSM $<$ pT3, number ( $\%$ on 115 patients) & $2(3.6 \%)$ & $9(15.5 \%)$ & 0.031 \\
\hline $\mathrm{PSM} \geq \mathrm{pT} 3$, number ( $\%$ on 47 patients) & $10(41.7 \%)$ & $8(34.8 \%)$ & 0.393 \\
\hline PSM nerve-sparing procedure, number ( $\%$ on 88 patients) & $5(10.9 \%)$ & $7(16.7 \%)$ & 0.429 \\
\hline Multifocal PSM, number ( $\%$ out of 29 patients with PSM) & $6(50.0 \%)$ & $9(53.0 \%)$ & 0.876 \\
\hline \multicolumn{4}{|l|}{ Site of PSM, number ( $\%$ out of 29 patients with PSM) } \\
\hline Apical & $8(66.7 \%)$ & $4(23.5 \%)$ & 0.020 \\
\hline Posterior & $8(66.7 \%)$ & $10(58.8 \%)$ & 0.668 \\
\hline Lateral & $2(16.7 \%)$ & $5(29.4 \%)$ & 0.430 \\
\hline Anterior & $3(25.0 \%)$ & $5(29.4 \%)$ & 0.793 \\
\hline Base & $1(8.3 \%)$ & $2(11.8 \%)$ & 0.765 \\
\hline \multicolumn{4}{|l|}{ Post-operative PSA (ng/mL), median (IQR) } \\
\hline 1 month & $0.015(0.01-0.04)$ & $0.02(0.01 .0 .05)$ & 0.484 \\
\hline 3 months & $0.01(0.01-0.04)$ & $0.018(0.005-0.04)$ & 0.913 \\
\hline 6 months & $0.01(0.01-0.04)$ & $0.021(0.01-0.04)$ & 0.378 \\
\hline
\end{tabular}

with the existing literature is fairly limited, since the only available randomized trial on DVC management enrolled a lower number of cases (30 vs 30 cases), involved one single expert surgeon, and dealt with purely laparoscopic prostatectomy [10]. Thus, given the technical differences between laparoscopy and robotics the major finding - an advantage on early continence recovery for d-DVC — cannot be directly translated to our setting. Also the literature specifically dealing with this issue of DVC management in robotics is poor and restricted to three retrospective comparative studies, with single-surgeon cohorts in size comparable to ours: Lei and coll [11] compared 303 s-DVC and 240 d-DVC, Woldu and coll [12] 118 s-DVC and 126 d-DVC and Otsuki and coll [13] 115 s-DVC and 62 d-DVC. The results on morbidity were consistent among all the studies and confirmed the safety of a delayed approach: a higher EBL in d-DVC group was uniformly reported, generally reaching statistical significance $[11,13]$, but no difference was noted in clinical course, transfusion and complication rates. Functional outcomes were similar, too: except for the study from Lei and coll [11] that found a difference in the 6-month continence rate, higher for d-DVC approach (61.4 vs 39.6\%, definition $0 \mathrm{pad} /$ day), no differences in continence or potency recovery were observed, at any time point. These results, on the whole, confute the assumption that an early standard approach could negatively influence functional recovery due to a damage to the sphincter or neurovascular bundles. This hypothesis, claimed considering that the ventral and more prominent portion of the striated sphincter could be entrapped by an inappropriate suture of the DVC [2, 24], probably should be renegotiated for robotic surgery. Indeed only studies on purely laparoscopic prostatectomy showed worse early continence rates for the standard approach [10, $25,26]$, probably in virtue of a less accurate suture. 
The more debatable finding of our study concerns the data on PSM, also considering the inconsistency with other published data. Indeed, Lei [11] found no differences in overall and apical PSM rates, Woldu [12] found no differences in apical PSM and Otsuki [13] found a significant higher overall PSM in d-DVC (26.1 vs $11.3 \%$ ), but similar involvement of the apex. First of all, we must acknowledge that the design of our trial was not powered to detect differences in PSM rate and, therefore, no definitive conclusions should be reached on this. At the same time, it should be remarked that the randomized and prospective design probably mitigated biases, by generating two groups with equal clinical, surgical and pathological features. Therefore, the conclusions we reached were reasonably at least more solid than other studies. To attempt at a cautious explanation of our findings, we can hypothesize that the lower PSM rate in pT2 PCa for the s-DVC group could be related to a reduction in venous back-flow that aided the dissection from bladder neck to the apex. On the other hand, the differences in the prevalence of apical margins could be due to an improved precision during apical dissection given by the higher mobility of prostate in case of delayed ligation of the DVC. Even if such considerations require to be confirmed by larger series to balance the possible effects due to chance, they could constitute the base on which to tailor surgical strategy according to the localization of $\mathrm{PCa}$ at pre-operative framework. In particular, the d-DVC could be preferred in case of suspicious apical involvement, whereas the s-DVC when PCa involves other sites of the gland. Despite the lack of advantages at randomized comparison, at now our preference is reserved to a delayed modified approach, suturing the DVC after its section but before apical dissection and urethral detachment, to favor a selective ligature.

Finally, we believe that our results could be reproduced at the majority of institutions due to the involvement of two different surgeons with not extensive, even well established, experience and not of a single highly experienced surgeon.

The major limitation of the study is related to the estimation of sample size on EBL. So that, our findings on positive margins, continence and potency should be considered less definitive.

\section{Conclusion}

The results from the present trial suggest that both standard and delayed approaches to the DVC in RARP are safe with equal functional outcomes. The standard approach seems to expose to a lower risk of PSM in organ-confined disease at all sites, whereas the delayed approach should benefit a lower risk of positive apical margins.
Funding This research did not receive any specific grant from funding agencies in the public, commercial, or not-for-profit sectors.

\section{Compliance with ethical standards}

Conflict of interest All the authors declare that they have no conflict of interest.

\section{References}

1. Walsh PC (1988) Radical retropubic prostatectomy with reduced morbidity: an anatomic approach. NCI Monogr 7:133-137

2. Ganzer R, Stolzenburg JU, Neuhaus J, Weber F, Burger M, Bründl J (2014) Is the striated urethral sphincter at risk by standard suture ligation of the dorsal vascular complex in radical prostatectomy? An anatomic study. Urology 84:1453-1458. https://doi. org/10.1016/j.urology.2014.06.092

3. Leow JJ, Chang SL, Meyer CP, Wang Y, Hanske J, Sammon JD, Cole AP, Preston MA, Dasgupta P, Menon M, Chung BI, Trinh QD (2016) Robot-assisted versus open radical prostatectomy: a contemporary analysis of an all-payer discharge database. Eur Urol 70:837-845. https://doi.org/10.1016/j.eururo.2016.01.044

4. Ilic D, Evans SM, Allan CA, Jung JH, Murphy D, Frydenberg M (2017) Laparoscopic and robot-assisted vs open radical prostatectomy for the treatment of localized prostate cancer: a Cochrane systematic review. BJU Int https://doi.org/10.1111/bju.14062. (Epub ahead of print)

5. Antonelli A, Sodano M, Peroni A, Mittino I, Palumbo C, Furlan M, Carobbio F, Tardanico R, Fisogni S, Simeone C (2017) Positive surgical margins and early oncological outcomes of robotic vs open radical prostatectomy at a medium case-load institution. Minerva Urol Nefrol 69:63-68. https://doi.org/10.23736/S0393 $-2249.16 .02518-2$

6. Yaxley JW, Coughlin GD, Chambers SK, Occhipinti S, Samaratunga H, Zajdlewicz L, Dunglison N, Carter R, Williams S, Payton DJ, Perry-Keene J, Lavin MF, Gardiner RA (2016) Robot-assisted laparoscopic prostatectomy versus open radical retropubic prostatectomy: early outcomes from a randomised controlled phase 3 study. Lancet 388:1057-1066. https://doi.org/10.1016/S0140 $-6736(16) 30592-\mathrm{X}$

7. Nossiter J, Sujenthiran A, Charman SC, Cathcart PJ, Aggarwal A, Payne H, Clarke NW, van der Meulen J (2018) Robot-assisted radical prostatectomy vs laparoscopic and open retropubic radical prostatectomy: functional outcomes 18 months after diagnosis from a national cohort study in England. Br J Cancer 118:489 494. https://doi.org/10.1038/bjc.2017.454

8. Tüfek I, Atuğ F, Argun B, Keskin S, Obek C, Coşkuner E, Kural AR (2012) The use of a bulldog clamp to control the dorsal vein complex during robot-assisted radical prostatectomy. J Endourol 26:1605-1608. https://doi.org/10.1089/end.2012.0153

9. Wu SD, Meeks JJ, Cashy J, Perry KT, Nadler RB (2010) Suture versus staple ligation of the dorsal venous complex during robotassisted laparoscopic radical prostatectomy. BJU Int 106:385-390. https://doi.org/10.1111/j.1464-410X.2009.09146.x

10. Porpiglia F, Fiori C, Grande S, Morra I, Scarpa RM (2009) Selective versus standard ligature of the deep venous complex during laparoscopic radical prostatectomy: effects on continence, blood loss, and margin status. Eur Urol 55:1377-1383. https://doi. org/10.1016/j.eururo.2009.02.009

11. Lei Y, Alemozaffar M, Williams SB, Hevelone N, Lipsitz SR, Plaster BA, Amarasekera CA, Ulmer WD, Huang AC, Kowalczyk $\mathrm{KJ}$, Hu JC (2010) Athermal division and selective suture ligation of the dorsal vein complex during robot-assisted laparoscopic 
radical prostatectomy: description of technique and outcomes. Eur Urol 59:235-243. https://doi.org/10.1016/j.eururo.2010.08.043

12. Woldu SL, Patel T, Shapiro EY, Bergman AM, Badani KK (2013) Outcomes with delayed dorsal vein complex ligation during robotic assisted laparoscopic prostatectomy. Can J Urol 20:7079-7083

13. Otsuki H, Nakamura K, Kuwahara Y, Tsukamoto T (2015) Modifications for controlling the dorsal vein complex in robotic-assisted laparoscopic radical prostatectomy. Nihon Hinyokika Gakkai Zasshi 106:7-11

14. Schulz KF, Altman DG, Moher D, CONSORT Group (2010) CONSORT 2010 statement: updated guidelines for reporting parallel group randomised trials. BMJ 23(340):c332. https://doi. org/10.4103/0976-500X.72352

15. Charlson ME, Pompei P, Ales KL, MacKenzie CR (1987) A new method of classifying prognostic comorbidity in longitudinal studies: development and validation. J Chronic Dis 40:373-383

16. Daley J, Khuri SF, Henderson W, Hur K, Gibbs JO, Barbour G, Demakis J, Irvin G, Stremple JF, Grover F, McDonald G, Passaro E, Fabri PJ, Spencer J, Hammermeister K, Aust JB, Oprian C (1997) Risk adjustment of the postoperative mortality rate for the comparative assessment of the quality of surgical care: results of the National Veterans Affairs Surgical Risk Study. J Am Coll Surg 185:315-327

17. Gandaglia G, Fossati N, Zaffuto E, Bandini M, Dell'Oglio P, Bravi CA, Fallara G, Pellegrino F, Nocera L, Karakiewicz PI, Tian Z, Freschi M, Montironi R, Montorsi F, Briganti A (2017) Development and internal validation of a novel model to identify the candidates for extended pelvic lymph node dissection in prostate cancer. Eur Urol 72:632-640. https://doi.org/10.1016/j.eurur o.2017.03.049

18. D'Amico A, Altschuler M, Whittington R, Kao G, Malkowicz SB, Wein A (1993) The use of clinical parameters in an interactive statistical package to predict pathological features associated with local failure after radical prostatectomy for prostate cancer. Clin Perform Qual Health Care 1:219-222

19. Dindo D, Demartines N, Clavien PA (2004) Classification of surgical complications: a new proposal with evaluation in a cohort of 6336 patients and results of a survey. Ann Surg 240:205-213
20. Mitropoulos D, Artibani W, Biyani CS, Bjerggaard Jensen J, Rouprêt M, Truss M (2017) Validation of the Clavien-Dindo grading system in urology by the European Association of Urology guidelines ad hoc panel. Eur Urol Focus. https://doi.org/10.1016/j. euf.2017.02.014. (Epub ahead of print)

21. Epstein JI, Egevad L, Amin MB, Delahunt B, Srigley JR, Humphrey PA, Grading C (2016) The 2014 international society of urological pathology (ISUP) consensus conference on gleason grading of prostatic carcinoma: definition of grading patterns and proposal for a new grading system. Am J Surg Pathol 40:244-252. https://doi.org/10.1097/PAS.0000000000000530

22. Wei JT, Dunn RL, Litwin MS, Sandler HM, Sanda MG (2000) Development and validation of the expanded prostate cancer index composite (EPIC) for comprehensive assessment of health-related quality of life in men with prostate cancer. Urology 56:899-905

23. Rocco B, Coelho RF, Albo G, Patel VR (2010) Robot-assisted laparoscopic prostatectomy: surgical technique. Minerva Urol Nefrol 62:295-304

24. Walz J, Epstein JI, Ganzer R, Graefen M, Guazzoni G, Kaouk J, Menon M, Mottrie A, Myers RP, Patel V, Tewari A, Villers A, Artibani W (2016) A critical analysis of the current knowledge of surgical anatomy of the prostate related to optimisation of cancer control and preservation of continence and erection in candidates for radical prostatectomy: an update. Eur Urol 70:301-311. https ://doi.org/10.1016/j.eururo.2016.01.026

25. Hoshi A, Usui Y, Shimizu Y, Tomonaga T, Kawakami M, Nakajima N, Hanai K, Nomoto T, Terachi T (2013) Dorsal vein complex preserving technique for intrafascial nerve-sparing laparoscopic radical prostatectomy. Int J Urol 20:493-500. https://doi. org/10.1111/j.1442-2042.2012.03181.x

26. Xu P, Xu A, Chen B, Zheng S, Xu Y, Li H, Li B, Huang P, Zhang Y, Ge Y, Liu C (2017) Ligation-free technique for dorsal vascular complex control during laparoscopic radical prostatectomy: a single-center experience from China. World J Urol 35:395-402. https://doi.org/10.1007/s00345-016-1889-0 